\title{
Avian diversity in geothermal power plant areas: Case studies in Kamojang, Darajat, and Gunung Salak, West Java, Indonesia
}

\author{
TEGUH HUSODO ${ }^{1,2,3}$, KHEMAL PASHA MOCHTAN $^{3}$, SYA SYA SHANIDA ${ }^{3, \bullet}$, SYAHRAS FATHIN \\ AMINUDDIN $^{3}$, INDRI WULANDARI ${ }^{1,2,3}$, IRWANANDA SATRIA PUTRA ${ }^{4}$, ERRI NOVIAR MEGANTARA ${ }^{1,2,3}$ \\ ${ }^{1}$ Department of Biology, Faculty of Mathematics and Natural Sciences, Universitas Padjadjaran. Jl. Raya Bandung-Sumedang Km 21, Jatinangor, \\ Sumedang 45363, West Java, Indonesia \\ ${ }^{2}$ Program in Environmental Science, School of Graduates, Universitas Padjadjaran. Jl. Sekeloa, Coblong, Bandung 40134, West Java, Indonesia \\ ${ }^{3}$ Center of Environment and Sustainable Science, Directorate of Research, Community Services and Innovation, Universitas Padjadjaran. Jl. Raya \\ Bandung-Sumedang Km 21, Jatinangor, Sumedang 45363, West Java, Indonesia. Tel. +62-22-7796412 ext. 104, Fax. +62-22-7794545, `email: \\ syasyashanida@gmail.com \\ ${ }^{4}$ Indonesia Power, Unit Pembangkitan dan Jasa Pembangkitan Kamojang, Komplek Perumahan PLTP Kamojang 44101, Garut, West Java, Indonesia
}

Manuscript received: 28 December 2019. Revision accepted: 14 February 2020.

\begin{abstract}
Husodo T, Mochtan KP, Shanida SS, Aminuddin SF, Wulandari I, Putra IS, Megantara EN. 2020. Avian diversity in geothermal power plant areas: Case studies in Kamojang, Darajat, and Gunung Salak, West Java, Indonesia. Biodiversitas 21: 10491059. Land cover changes that occur in the development of industrial areas have impact on the quality of biophysical and socio-economic environments. This study aimed to investigate the diversity of birds (avifauna) in three geothermal power plant areas in West Jawa Province, Indonesia (i.e., Kamojang, Darajat, and Gunung Salak). Transect and spotlighting methods were conducted in this study. We found that areas around geothermal power plant in Gunung Salak had the highest diversity of bird species with 105 species from 43 families, followed by Kamojang with 82 species from 34 families, and Darajat with 80 species from 40 families. In total, there were 134 species from 51 families in which two species are Endangered (EN) under the IUCN Red List category, five are endemic to Java, 11 are protected by CITES Appendix II and 14 are protected by national regulations. The presence of some birds was an indicator of ecosystem condition and functioning, including indicator of food chain, quality of water, quality of natural habitat, condition of riparian habitat, and the condition of open or disturbed habitat. Some birds were also associated with particular habitats, such as natural forests, riparians, a combination of natural forests and open habitats, tea plantations, and craters. The results of this study can be used as baseline information about the state of the surrounding environment in the three geothermal power plant areas and as a reference in biodiversity management in the future.
\end{abstract}

Keywords: Avifauna, Geothermal Power Plants, indicator

Abbreviation: UPJP: Generation Unit and Generation Service; PLTP: Geothermal Power Plant

\section{INTRODUCTION}

Changes or differences in habitat affect animal diversity and distribution including birds or avifauna (Altaf et al., 2018). For example, a gradient from arable fields to forest landscape showed different avifauna species (Kosickia and Chylarecki 2012). Birds are often recognized as bioindicator for the state of environment because of their strong sensitivity to ecosystem changes. As bioindicators, the presence of birds could explain to what extent human activities have changed the habitat quality and how it affects biodiversity in a more general context. In addition, birds have social, economic, and cultural functions such as for food, pet, and source of folk songs and tales (Teixeira et al. 2014; Iskandar and Iskandar 2015; Partasasmita et al. 2016), as shown in countries that hold their traditions such as Indonesia (Krisanti et al. 2017).

According to Frederick et al. (2009), birds are also good indicators for ecosystem restoration program. There is broad consensus among experts that birds are the most suitable biological indicators for monitoring the health of an ecosystem as they are eye-catching and sensitive towards environmental changes (Khan et al. 2013). Ecologically, birds play tremendous importance because of their key roles as pollinators and agents of seed dispersal (Bibi and Ali 2013). Simple biological monitoring through bird surveys can demonstrate the condition of living systems in a landscape of interest. Such knowledge is more direct and integrative than information that comes from chemical or physical testings, which are generally more rigorous, expensive, time-consuming, and labor-intensive (Khan et al. 2013).

Industrial activities generally have negative impacts on the ecosystem and biodiversity in a landscape (Budiharta et al., 2018). As such, some industrial entities develop strategies to manage and conserve biodiversity. For example, an area for biodiversity conservation set aside within an industrial complex (Budiharta et al., 2010; Fiqa et al., 2019; Sudrajat and Putro, 2019). Another strategy includes minimizing the impacts of industrial activities by implementing environmentally friendly operation and production systems. This is particularly important for industry working on energy sector to produce eco-friendly energy, to maintain clean and green environment, and to mitigate impacts on biodiversity in which all of them are aimed to achieve environmental sustainability. Also, recently, some governments including Indonesia have 
enacted regulations regarding environmental management in industrial sector in which one of the issues highlights biodiversity conservation (Act No. 4 of 1982).

Biodiversity monitoring using birds as bioindicators can be used to assess the impacts of activities of industry working on energy sector including in geothermal power plants. Although geothermal power plant is considered as an eco-friendly energy-generating powerhouse compared to other sources (e.g. coal-generated power plant), it is still important to know whether its operation affects biodiversity. This is because there are some potential environmental problems that arise, such as land cover changes caused by forest conversion during the development of geothermal power plants which have impacts on the quality of the biophysical and socio-economic environment in the surrounding areas.

This study aimed to investigate the diversity of birds (avifauna) in three geothermal power plant areas in West Jawa Province, Indonesia (i.e. Kamojang, Darajat, and Gunung Salak). The results of this study can be used as baseline information about the state of the surrounding environment in the three geothermal power plant areas, especially in the context of biodiversity, which is currently experiencing threats or disturbances. Further, the information can also be used as a reference in biodiversity management in the future

\section{MATERIALS AND METHODS}

\section{Study area and period}

The study was conducted in Kamojang (July 2017), Darajat (May 2018), and Gunung Salak (July 2018), all are located in West Java Province, Indonesia. Administratively, Darajat is located in Padaawas Village,
Pasirwangi Sub-district, Garut District. The land cover types in Darajat consisted of craters, riparian, lakes, natural forests, and existing buildings. Kamojang is located in Laksana Village, Ibun sub-district, Bandung District. The land cover types in Kamojang consisted of artificial parks, horticulture fields, plantations, pine forests, riparian, shrubs, swamps, natural forests, and existing buildings. Gunung Salak is located in Purwabakti Village, Pamijahan Sub-district, Bogor District. Gunung Salak is within the Halimun-Gunung Salak National Park. The land cover types in Gunung Salak consisted of natural forests, riparian, craters, shrubs, tea plantations, settlements, and existing buildings.

\section{Procedures}

Transect and spotlighting

Data was collected using transect and spotlighting methods. The transect method was carried out every day in the morning starting at $05.30-11.00$ a.m. and in the afternoon starting at 02.00-05.00 p.m. Data collection was conducted on foot $( \pm 2 \mathrm{~km} /$ hour$)$ by recording avifauna species either they were visible and/or they were only audible. This transect method was based on the proportion of the area observed and the time available for observation.

The spotlighting method was carried out to record nocturnal species and conducted at 07.00-11.00 p.m. using flashlight and headlamp. Night-watching was repeated on the track that has been traversed in the morning or carried out at locations that were considered to have the potential in encountering the avifauna.

\section{Data analysis}

Data was analyzed qualitatively. The species were identified using MacKinnon and Phillipps (1993).

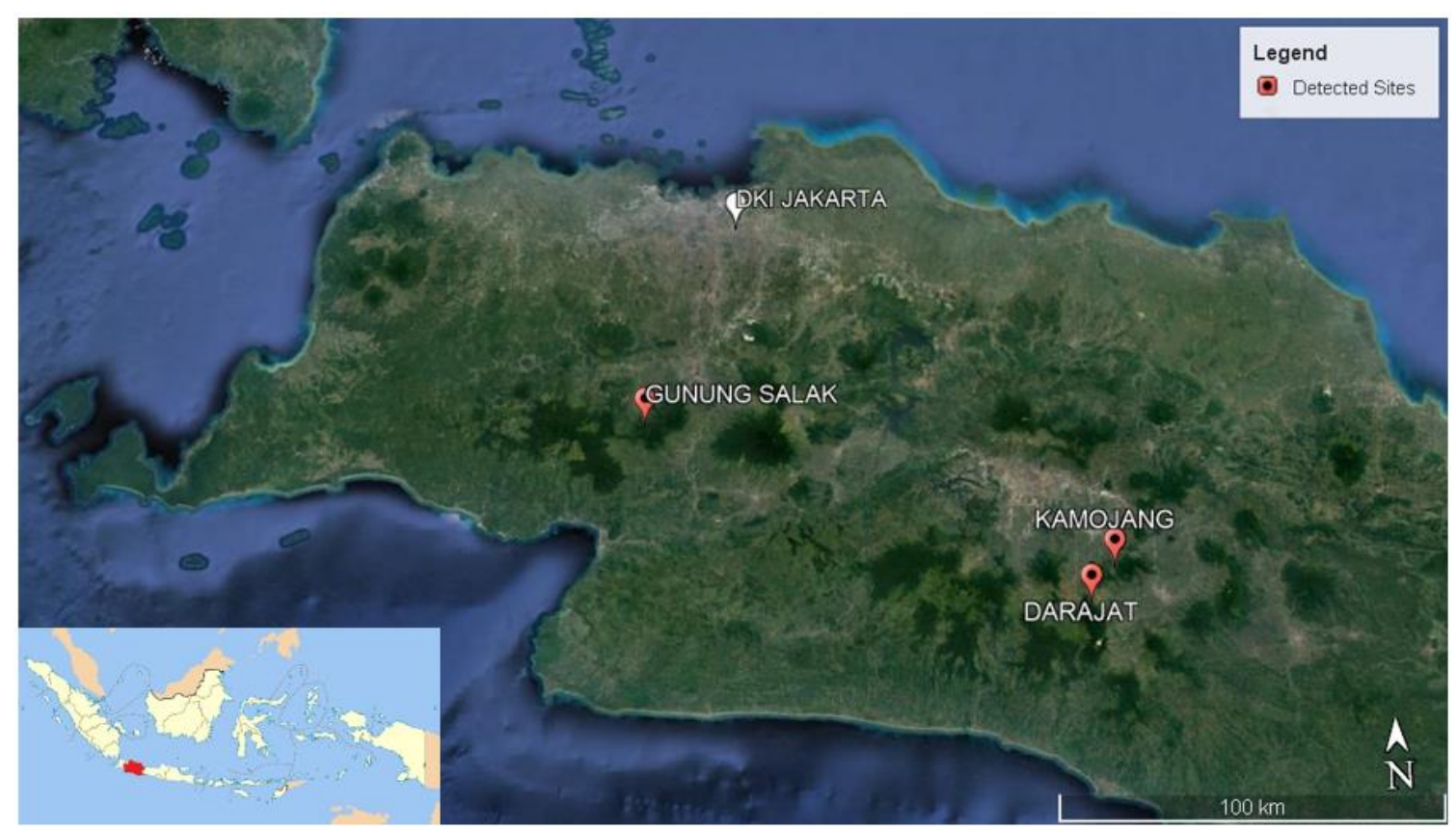

Figure 1. Study areas in West Java, Indonesia: Darajat, Kamojang and Gunung Salak. Map source: Google Earth (2019) 


\section{RESULTS AND DISCUSSION}

\section{Avifauna diversity}

A total of 134 species from 51 families were recorded in Kamojang, Darajat and Gunung Salak (Table 1). Muscicapidae was the most recorded family in this study. In Kamojang there were 82 species from 34 families while in Darajat there were 80 species from 40 families, and in Gunung Salak there were 105 species from 43 families. There were 49 species found in all study locations. Based on these results, it is assumed that those three locations had the availability of food and shelter to meet the needs of birds. Also, it can be assumed that these species had a high tolerance of human disturbances.

\section{Conservation status}

Among 134 species, there are two species with EN (Endangered) conservation status under the IUCN Red List, eight species with NT (Near Threatened), and three species with VU (Vulnerable) (Table 1). Of 49 species found in three locations, one of them has Vulnerable status (i.e. Cochoa azurea) while other species are under the Least Concern status. In addition, there are 11 species protected by CITES in the Appendix II category and 14 species protected by national protection regulations.

Species with EN conservation status are Chloropsis cochinchinensis and Nisaetus bartelsi. Species with NT conservation status are Caprimulgus pulchellus, Chrysophlegma mentale, Eurylaimus javanicus, Laniellus albonotatus, Loriculus pusillus, Megalaima javensis, Prinia familiaris, and Pycnonotus bimaculatus. Species with VU conservation status are Cochoa azurea, Otus angelinae, and Rhyticeros undulatus.

Families with the entire species are protected by CITES in all three locations are Accipitridae, Bucerotidae, Falconidae, Psittacidae, and Strigidae. Within Accipitridae family, there were 5 species in which one species has EN conservation status, which is Nisaetus bartelsi (Javan hawk-eagle). This $N$. bartelsi is an endemic raptor of Java Island which is currently severely threatened by both wildlife trade and habitat loss (Nursamsi et al. 2018).

\section{Birds as indicators in ecosystem}

In the three study areas of geothermal power plant complex, several species were found as ecosystem indicators, including Ictinaetus malaiensis and Spilornis cheela which acted as indicators of habitat quality. This species is a predator that controls the food chain in ecosystem, resulting in a balance of ecosystem. While carnivorous birds that hunt and prey on small animals, such as rodents, would keep the population of the vermin under control, leaving the food chain structures balanced through this act of predation (Praptiwi et al. 2019).

Alcippe pyrrhoptera and Psilopogon armilaris are indicators of natural habitats quality. The existence of this species is commonly found in natural forests and eating forest fruits, such as walen (Ficus ribes), manganese (Mallotus sp.), and fig (Ficus rostrata). Another species,
Arachnothera affinis, is known as a natural pollinator. The existence of these species indicates that in the study locations natural pollination and seed dispersal still occur. According to Praptiwi et al. (2019), when birds ingest fruits from fruit-bearing trees, they would leave the seeds undigested, excreting them to the ground and leaving them ready for germination. Birds that consume pollen from flowering plants would carry them along with pollens, transporting and inadvertently distributing the pollens into nearby flowers, causing them to be pollinated.

Enicurus leschenaulti is an indicator of riparian habitat quality. This species is often found in river with but not swift streams. This species has also been found looking for leaf litter on river rocks to make nests. Halcyon cyanoventris from the Alcedinidae Family is an indicator of water quality. This species indicates that the condition of the aquatic ecosystems in these three regions is still good.

Megalurus palustris, Lonchura leucogastroides, Pycnonotus aurigaster, and Passer montanus are indicators of open and disturbed habitat. Suryawan et al. (2017) said that Passer montanus is commonly found around the housing of residents in villages. These species have a high tolerance level to the presence and interference of humans, indicated by their ability to make nests in residential and development areas. Although they were found in disturbed habitats, if the site can still provide resources for the species, the site will certainly continue to be utilized for their survival.

Based on the description above, the presence of some species of birds as bio-indicators suggests the quality of ecosystems around the three geothermal power plant complexes in West Java. These ecosystem indicators include indicator of food chain, good water quality, quality of natural habitats, riparian habitats, and open or disturbed habitats. Birds as one of the most visible residents of the threatened areas hold a key role in the functioning of the ecosystem. They perform services essential for the regulation of ecosystem health, such as seeds dispersal, pollination of tropical plants and pest control (Philpott et al. 2009, Sekercioglu 2012). An important point to consider concerning its roles in maintaining the ecosystem balance is that whether the species composition found on each terrain is reasonable (Krisanti et al. 2017).

\section{Bird diversity and habitat characteristics Natural forest}

Species found in the core of natural forest were Megalaima javensis, Psilopogon corvinus, Rhyticeros undulatus, Ducula badia, Macropygia emiliana, Macropygia ruficeps, Laniellus albonotatus and Macropygia unchall. These species were often found in tall and large trees for their nesting place and fruit source. Similar to Megalaima armillaris, these species are indicators of natural habitat. For these species, forest is a vital habitat that provides abundant food, water, and shelters to sustain their life. The absence of forests might lead to their extinction (Krisanti et al. 2017). 
Table 1. Bird diversity in three geothermal power plant complexes in West Java

\begin{tabular}{|c|c|c|c|c|c|c|c|c|c|}
\hline \multirow{2}{*}{ Family | Species } & \multirow{2}{*}{ English Name } & \multirow{2}{*}{ Local Name } & \multicolumn{3}{|c|}{ Locations } & \multicolumn{3}{|c|}{ Conservation Status } & \multirow{2}{*}{ Endemic } \\
\hline & & & KMJ & DRJ & GS & RI & IUCN & CITES & \\
\hline \multicolumn{10}{|l|}{ Acanthizidae } \\
\hline Gerygone sulphurea (Wallace, 1864) & Golden-bellied Gerygone & Rametuk Laut & & 1 & 1 & & $\mathrm{LC}$ & & \\
\hline \multicolumn{10}{|l|}{ Accipitridae } \\
\hline Ictinaetus malaiensis (Temminck, 1822) & Black Eagle & Elang Hitam & 1 & 1 & 1 & + & $\mathrm{LC}$ & II & \\
\hline Nisaetus bartelsi (Stresemann, 1924) & Javan Hawk-eagle & Elang Jawa & & & 1 & + & EN & II & $\mathrm{J}$ \\
\hline Nisaetus cirrhatus (Gmelin, 1788) & Crested Hawk-Eagle & Elang Brontok & 1 & & 1 & + & $\mathrm{LC}$ & II & \\
\hline Pernis ptilorhynchus (Temminck, 1821) & Oriental Honey-buzzard & Sikep-madu Asia & 1 & & & + & $\mathrm{LC}$ & II & \\
\hline Spilornis cheela (Latham, 1790) & Crested Serpent Eagle & Elang-ular Bido & 1 & 1 & 1 & + & $\mathrm{LC}$ & II & \\
\hline \multicolumn{10}{|l|}{ Aegithalidae } \\
\hline Psaltria exilis (Temminck, 1836) & Pygmy Tit & Cerecet Jawa & 1 & 1 & 1 & + & $\mathrm{LC}$ & & $\mathrm{J}$ \\
\hline \multicolumn{10}{|l|}{ Aegithinidae } \\
\hline Aegithina tiphia (Linnaeus, 1758) & Common Iora & Cipoh Kacat & & 1 & & & $\mathrm{LC}$ & & \\
\hline \multicolumn{10}{|l|}{ Alcedinidae } \\
\hline Alcedo meninting (Horsfield, 1821) & Blue-eared Kingfisher & Raja-udang Meninting & 1 & & & & $\mathrm{LC}$ & & \\
\hline Halcyon cyanoventris (Vieillot, 1818) & Javan Kingfisher & Cekakak Jawa & 1 & 1 & & & $\mathrm{LC}$ & & $\mathrm{J}$ \\
\hline Todiramphus chloris (Boddaert, 1783) & Collared Kingfisher & Cekakak Sungai & 1 & 1 & & & $\mathrm{LC}$ & & \\
\hline \multicolumn{10}{|l|}{ Apodidae } \\
\hline Apus affinis (Gray, 1830) & Little Swift & Kapinis Rumah & & & 1 & & $\mathrm{LC}$ & & \\
\hline Apus nipalensis (Hodgson, 1836) & House Swift & Kapinis Rumah & 1 & 1 & & & $\mathrm{LC}$ & & \\
\hline Collocalia esculenta (Linnaeus, 1758) & Glossy Swiftlet & Walet Linci & 1 & 1 & 1 & & $\mathrm{LC}$ & & \\
\hline \multicolumn{10}{|l|}{ Ardeidae } \\
\hline Ixobrychus cinnamomeus (Gmelin, 1789) & Cinnamon Bittern & Bambangan Merah & 1 & & & & $\mathrm{LC}$ & & \\
\hline \multicolumn{10}{|l|}{ Artamidae } \\
\hline Artamus leucorynchus (Linneaus, 1771) & Artamus Leucoryn & Kekep Babi & 1 & 1 & 1 & & $\mathrm{LC}$ & & \\
\hline \multicolumn{10}{|l|}{ Bucerotidae } \\
\hline Rhyticeros undulatus (Shaw, 1811) & Rhinoceros Hornbill & Julang Emas & & & 1 & + & VU & II & \\
\hline \multicolumn{10}{|l|}{ Campephagidae } \\
\hline Coracina javensis (Horsfield, 1821) & Large Cuckooshrike & Kepudang-sungu Jawa & & 1 & 1 & & $\mathrm{LC}$ & & $\mathrm{J}, \mathrm{B}$ \\
\hline Coracina larvata (S. Müller, 1843) & Sunda Cuckooshrike & Kepudang-sungu Gunung & & 1 & & & $\mathrm{LC}$ & & \\
\hline Lalage fimbriata (Temminck, 1824) & Lesser Cuckooshrike & Kepudang-sungu Kecil & & 1 & 1 & & $\mathrm{LC}$ & & \\
\hline Pericrocotus cinnamomeus (Linnaeus, 1766) & Small Minivet & Sepah Kecil & & & 1 & & $\mathrm{LC}$ & & \\
\hline Pericrocotus miniatus (Temminck, 1822) & Sunda Minivet & Sepah Gunung & 1 & & 1 & & $\mathrm{LC}$ & & $\mathrm{S}, \mathrm{J}$ \\
\hline \multicolumn{10}{|l|}{ Caprimulgidae } \\
\hline Caprimulgus pulchellus (Salvadori, 1879) & Salvadori's Nightjar & Cabak Gunung & & & 1 & & NT & & $\mathrm{J}$ \\
\hline \multicolumn{10}{|l|}{ Cettidae } \\
\hline Horornis vulcanius (Blyth, 1870) & Sunda Bush-warbler & Ceret Gunung & 1 & 1 & & & $\mathrm{LC}$ & & \\
\hline
\end{tabular}


Cisticolidae

Cisticola juncidis (Rafinesque, 1810) Orthotomus ruficeps (Lesson, 1830)

Orthotomus sepium (Horsfield, 1821)

Orthotomus sutorius (Pennant, 1769)

Prinia familiaris (Horsfield, 1821)

Prinia flaviventris (Delessert, 1840)

Chloropseidae

Chloropsis cochinchinensis (Gmelin, 1789)

\section{Columbidae}

Chalcophaps indica (Linnaeus, 1758)

Ducula badia (Raffles, 1822)

Macropygia emiliana (Bonaparte, 1854)

Macropygia ruficeps (Temminck, 1834)

Macropygia unchall (Wagler, 1827)

Ptilinopus porphyreus (Temminck, 1823)

Spilopelia chinensis (Scopoli, 1786)

\section{Cuculidae}

acomantis merulinus (Scopoli, 1786)

Cacomantis sepulcralis (Vigors \& Horsfield, 1826)

Centropus bengalensis (Gmelin, 1788)

Cuculus saturatus (Blyth, 1843)

Phaenicophaeus curvirostris (Shaw, 1810)

Surniculus lugubris (Horsfield, 1821)

Zanclostomus javanicus (Horsfield, 1821)

\section{Dicaeidae}

Dicaeum concolor (Jerdon, 1840)

Dicaeum sanguinolentum (Temminck, 1829)

Dicaeum trigonostigma (Scopoli, 1786)

Dicaeum trochileum (Sparrman, 1789)

Prionochilus percussus (Temminck \& Laugier, 1826)

\section{Dicruridae}

Dicrurus leucophaeus (Vieillot, 1817)

Dicrurus macrocercus (Vieillot, 1817)

Dicrurus remifer (Temminck, 1823)

\section{Estrildidae}

Erythrura hyperythra (Reichenbach, 1863)

Erythrura prasina (Sparrman, 1788)

Lonchura leucogastroides (Horsfield \& Moore, 1856)

Lonchura maja (Linnaeus, 1766)

Lonchura punctulata (Linnaeus, 1758)

Eurylaimidae

Eurylaimus javanicus (Horsfield, 1821)
Zitting Cisticola

Ashy Tailorbird

Olive-backed Tailorbird

Common Tailorbird

Bar-winged Prinia

Yellow-bellied Prinia

Javan Leafbird

Grey-capped Emerald Dove

Mountain Imperial-pigeon

Ruddy Cuckoo-dove

Little Cuckoo-dove

Barred Cuckoo-dove

Pink-headed Fruit-dove

Eastern Spotted Dove

Plaintive Cuckoo

Brush Cuckoo

Lesser Coucal

Oriental Cuckoo

Chestnut-breasted Malkoha

Square-tailed Drongo-cuckoo

Red-billed Malkoha

Nilgiri Flowerpecker

Blood-breasted Flowerpecker

Orange-bellied Flowerpecker

Scarlet-headed Flowerpecker

Crimson-breasted Flowerpecker

Ashy Drong

Black Drongo

Lesser Racquet-tailed Drongo

Tawny-breasted Parrotfinch

Pin-tailed Parrotfinch

Javan Munia

White-headed Munia

Scaly-breasted Munia

Javan Broadbill
Cici Padi

Cinenen Kelabu

Cinenen Jawa

Cinenen Pisang

Prenjak Jawa

Prenjak Rawa

Cica-daun Sayap-biru

Delimukan Zambrud

Pergam Gunung

Uncal Buau

Uncal Koran

Uncal Loreng

Walik Kepala ungu

Tekukur Biasa

Wiwik Kelabu

Wiwik Uncuing

Bubut Alang-alang

Kangkok Ranting

Kadalan Birah

Kedasi Hitam

Kadalan Kembang

Cabai Polos

Cabai Gunung

Cabai Bunga-api

Cabai Jawa

Pentis Pelangi

Srigunting Kelabu

Srigunting Hitam

Srigunting Bukit

Bondol Hijau-dada Merah

Bondol-hijau Binglis

Bondol Jawa

Bondol Haji

Bondol Peking

Sempur Hutan Rimba 


\section{Falconidae}

Falco moluccensis (Bonaparte, 1850)

Microhierax fringillarius (Drapiez, 1824)

\section{Hirundinidae}

Cecropis daurica (Linnaeus, 1771)

Cecropis striolata (Temminck \& Schlegel, 1847)

Hirundo tahitica (Gmelin, 1789)

Laniidae

Lanius schach (Linnaeus, 1758)

\section{Leiotrichidae}

Alcippe pyrrhoptera (Bonaparte, 1850)

Laniellus albonotatus (Lesson, 1832

Locustellidae

Megalurus palustris (Horsfield, 1821)

\section{Megalaimidae}

Megalaima armillaris (Temminck, 1821)

Megalaima javensis (Horsfield, 1821)

Megalaima lineata (Vieillot, 1816)

Psilopogon corvinus (Temminck, 1831)

\section{Muscicapidae}

Brachypteryx leucophris (Temminck, 1828)

Cyornis unicolor (Blyth, 1843)

Enicurus leschenaulti (Vieillot, 1818)

Enicurus velatus (Temminck, 1822)

Eumyias indigo (Horsfield, 1821)

Ficedula hyperythra (Blyth, 1843)

Ficedula mugimaki (Temminck, 1815)

Ficedula westermanni (Sharpe, 1888)

Muscicapa dauurica (Pallas, 1811)

Muscicapa sibirica (Gmelin, 1789)

Myophonus glaucinus (Temminck, 1823)

\section{Nectariniidae}

Aethopyga eximia (Horsfield, 1821)

Aethopyga siparaja (Raffles, 1822)

Arachnothera affinis (Horsfield, 1821)

Arachnothera longirostra (Latham, 1790)

Arachnothera robusta (Muller \& Schlegel, 1845)

Chalcoparia singalensis (Gmelin, 1789)

Cinnyris jugularis (Linneaus, 1766)

\section{Paridae}

Parus major (Linnaeus, 1758)

Passeridae

Passer montanus (Linnaeus, 1758)
Spotted Kestrel

Black-thighed Falconet

Red-rumped Swallow

Striated Swallow

Tahiti Swallow

Long-tailed Shrike

Javan Fulvetta

Spotted Crocias

Striated Grassbird

Flame-fronted Barbet

Black-banded Barbet

Lineated Barbet

Brown-throated Barbet

Lesser Shortwing

Pale Blue-flycatcher

White-crowned Forktail

Sunda Forktail

Indigo Flycatcher

Snowy-browed Flycatcher

Mugimaki Flycatcher

Little Pied Flycatche

Asian Brown Flycatcher

Dark-sided Flycatcher

Javan Whistling-thrush

White-flanked Sunbird

Crimson Sunbird

Streaky-breasted Spiderhunter

Little Spiderhunter

Long-billed Spiderhunter

Ruby-cheeked Sunbird

Olive-backed Sunbird

Great Tit

Eurasian Tree Sparrow
Alap-alap Sapi

Alap-alap Capung

Layang-layang gua

Layang-layang Loreng

Layang-layang Batu

Bentet Kelabu

Wergan Jawa

Cica Matahari

Cica Koreng

Takur Tohtor

Takur Tulung-tumpuk

Takur Bultok

Takur Bututut

Cingcoang Coklat

Sikatan Biru-muda

Meninting Besar

Meninting Kecil

Sikatan Nion

Sikatan Bodoh

Sikatan Mugimaki

Sikatan Belang

Sikatan Bubik

Sikatan Sisi-gelap

Ciung Batu-kecil

Burung Madu Gunung

Burung-madu Sepah-raja

Pijantung Gunung

Pijantung Kecil

Pijantung Besar

Burung Madu Belukar

Burung Madu Sriganti

Gelatik Batu

Burung-gereja Erasia 
Pellorneidae

Malacocincla sepiaria (Horsfield, 1821)

Napothera epilepidota (Temminck, 1827)

Trichastoma pyrrogenys (Temminck, 1827)

\section{Phasianidae}

Arborophila javanica (Gmelin, 1789)

Coturnix chinensis (Linnaeus, 1766)

Gallus gallus (Linnaeus, 1758)

Phylloscopidae

Phylloscopus grammiceps (Strickland, 1849)

Phylloscopus trivirgatus (Strickland, 1849)

\section{Picidae}

Chrysocolaptes validus (Temminck, 1825)

Chrysophlegma mentale (Temminck, 1825)

Dendrocopos macei (Vieillot, 1818)

Dendrocopos moluccensis (Gmelin, 1788)

Sasia abnormis (Temminck, 1825)

Podargidae

Batrachostomus javensis (Horsfield, 1821)

\section{Psittacidae}

Loriculus pusillus (Gray, 1859)

\section{Pnoepygidae}

Pnoepyga pusilla (Hodgson, 1845)

\section{Pycnonotidae}

Alophoixus bres (Lesson, 1832)

Iole viridescens (Blyth, 1845)

Pycnonotus aurigaster (Vieillot, 1818)

Pycnonotus bimaculatus (Horsfield, 1821)

Pycnonotus goiavier (Scopoli, 1786)

\section{Rallidae}

Amaurornis phoenicurus (Pennant, 1769)

\section{Scotocercidae}

Phyllergates cuculatus (Temminck, 1836)

Tesia superciliaris (Bonaparte, 1850)

\section{Stenostiridae}

Culicicapa ceylonensis (Swainson, 1820)

\section{Rhipiduridae}

Rhipidura phoenicura (Müller, 1843)

\section{Sittidae}

Sitta azurea (Lesson, 1830)

Sitta frontalis (Swainson, 1820)

Strigidae

Otus angelinae (Finsch, 1912)

Otus lempiji (Horsfield, 1821)
Horsfield's Babbler

Eyebrowed Wren-babbler

Temminck's Babbler

Chestnut-bellied Partridge

Asian Blue Quail

Red Junglefowl

Javan Warbler

Mountain Warbler

Orange-backed Woodpecker

Javan Yellownape

Fulvous-breasted Woodpecker

Sunda Pygmy Woodpecker

Rufous Piculet

Horsfield's Frogmouth

Yellow-throated Parrot

Pygmy Cupwing

Brown-cheeked Bulbul

Olive Bulbul

Sooty-headed Bulbul

Orange-spotted Bulbul

Yellow-vented Bulbul

White-breasted Waterhen

Mountain Tailorbird

Javan Tesia

Grey-headed Canary-flycatche

Rufous-tailed Fantail

Blue Nuthatch

Velvet-fronted Nuthatch

Javan Scops-owl

Sunda Scops-owl
Pelanduk Semak

Berencet Berkening

Pelanduk Bukit

Puyuh-gonggong Jawa

Puyuh Batu

Ayam-hutan Merah

Cikrak Muda

Cikrak Daun

Pelatuk Kundang

Pelatuk Kumis-kelabu

Caladi Ulam

Caladi Tilik

Tukik Tikus

Paruh-kodok Jawa

Serindit Jawa

Berencet Kerdil

Empuloh Janggut

Brinji Gunung

Cucak Kutilang

Cucak Gunung

Merbah Cerukcuk

Kareo Padi

Cinenen Gunung

Tesia Jawa

Sikatan Kepala-abu

Kipasan Ekor-merah

Munguk Loreng

Munguk Beledu

Celepuk Jawa

Celepuk Reban
LC

C

$\mathrm{LC}$

NT

LC

LC

LC

LC

NT

LC

LC

LC

LC

LC

LC
LC

VU 


\section{Aplonis panayensis (Scopoli, 1786)}

Timaliidae

Cyanoderma melanothorax (Temminck, 1823)

Stachyris thoracica (Temminck, 1821)

Turdidae

Cochoa azurea (Temminck, 1824)

Geokichla sibirica (Pallas, 1776)

Turnicidae

Turnix suscitator (Gmelin, 1789)

Vangidae

Hemipus hirundinaceus (Temminck, 1822)

Vireonidae

Pteruthius aenobarbus (Temminck, 1836)

Pteruthius flaviscapis (Temminck, 1836)

\section{Zosteropidae}

Heleia javanica (Horsfield, 1821)

Zosterops japonicus (Temminck \& Schlegel, 1845)

Zosterops palpebrosus (Temminck, 1824)
Asian Glossy Starling

Crescent-chested Babbler

White-bibbed Babbler

Javan Cochoa

Siberian Thrush

Barred Buttonquai

Black-winged Flycatcher-shrike

Chestnut-fronted Shrike-babbler

Pied Shrike-babbler

Javan Grey-throated White-eye

Mountain White-eye

Oriental White-eye
Perling Kumbang

Tepus Pipi-perak

Tepus Leher Putih

Ciung-mungkal Jawa

Anis Siberia

Gemak Loreng

Jingjing Batu

Ciu Kunyit

Ciu Besar

Opior Jawa

Kacamata Gunung

Kacamata Biasa
1

1

1

$1 \quad 1 \quad 1$

$1 \quad 1 \quad 1$

$\begin{array}{ccccc} & 1 & 1 & + & \text { LC } \\ & & 1 & & \text { LC } \\ 1 & 1 & 1 & & \text { LC } \\ 82 & 80 & 105 & 14 & \end{array}$

Note: Conservation Status: RI: Regulation of the Minister of Environment and Forestry of the Republic of Indonesia No. P 106 on Protected Plant and Animal Species; IUCN: International

Union for Conservation of Nature, LC: Least Concern, NT: Near Threatened, VU: Vulnerable, EN: Endangered, CITES: Convention on International Trade in Endangered Species, I: Appendix

I, Appendix II, Appendix III; Locations: KMJ: Kamojang, DRJ: Darajat, GS: Gunung Salak. Endemic: J: Java Island, B: Bali Island, K: Kalimantan Island, S: Sumatera, Sw: Sulawesi Island. 
Other species found in natural forests were Pericrocotus miniatus, Tesia superciliaris, Ficedula westermanni, Sitta azurea, Culicicapa ceylonensis, Rhipidura phoenicura, Alcippe pyrrhoptera, Psaltria exilis, Cyanoderma melanothorax, and Hemipus hirundinaceus. Certain species can only be found in natural forests such as Coracina javensis and Chrysophlegma mentale. It is possible that these two species can be found in other ecosystems such as in lakes or riparians, as long as they still have natural stands and have relatively the same environmental conditions.

Woodpeckers such as Chrysocolaptes validus were often found in pristine natural forests with old vegetation stands. It is because this species often forages on the trunks of old trees with many insects. In addition, these old trees can be used as nesting sites. The existence of this species is an indication that this habitat still has the carrying capacity for this species to find food and nest.

The structural heterogeneity and high diversity of plant species provide niches for many species of birds, and therefore can support a great number of bird species sharing the same habitat. The diversity of vegetation is an important factor affecting bird species diversity in the secondary forests (Erniwati et al. 2016).

\section{Water habitats}

Species found around pond area were Dicaeum concolor, Prionochilus percussus, Cyornis unicolor and Psilopogon corvinus. This location had puddles, which were used by birds to cool their body. Other birds found in riparian were Pnoepyga pusilla, Ficedula westermanni, Alcippe pyrrhoptera, Dicrurus leucophaeus, Culcicapa ceylonensis, Tesia superciliaris, and Myophonus glaucinus.

Species found around lake were Caprimulgus pulchellus, Otus angelinae and Batrachostomus javensis. The presence of these species around lake is likely due to the presence of frogs, toads, and small mammals as their foods. Species that can be found around river area were Lonchura leucogastroides, Pycnonotus bimaculatus, Aethopyga eximia, Pteruthius aenobarbus, Brachypteryx leucophrys, Ficedula westermanni, and Pnoepyga pusilla. Species recorded in swampy areas were Lonchura leucogastroides, Todiramphus chloris, Megalurus palustris, and Nectaria jugularis.

\section{The combination of natural forest and open habitat}

Species that occupied open habitats directly adjacent to natural forests were Erythrura hyperythra, Ptilinopus porphyreus, Caprimulgus pulchellus, Phaenicophaeus curvirostris, Zanclostomus javanicus, Dicrurus macrocercus, Enicurus velatus, Muscicapa dauica, and Batcocomis durica. Species that often used forest edge were Pericrocotus cinnamomeus and Pericrocotus miniatus. The species that inhabited other open habitats, such as shrubs, were Orthotomus ruficeps and Orthotomus sepium. Other species that often found in open and disturbed habitats were Spilopelia chinensis, Centropus bengalensis, Surniculus lugubris, Lonchura leucogastroides, Lonchura punctulata, Hirundo tahitica, Megalurus palustris, Pycnonotus aurigaster, Pycnonotus goiavier, and Turnix suscitator.

\section{Tea plantation}

Specifically, in Gunung Salak, there were tea plantations with remnants of natural vegetation and a part of the tea plantations that directly adjacent to natural forests. Due to this unique landscape, there was also a uniqueness of avifauna that lived there in which some species were they that commonly found in natural habitats while others were they that often found in open or disturbed habitats.

The species commonly found in natural habitats and found in this location were Psaltria exilis and Loriculus pusillus. Psaltria exilis were found in natural areas directly adjacent to tea plantations, while Loriculus pusillus were found in forest edge areas and areas where natural stands were still present. Loriculus pusillus is an avifauna that often uses tall tree canopies to fly from one tree to another. Loriculus pusillus was observed to fly from natural spotting stands in the middle of a tea garden. This is partly due to the presence of natural stands that were in a period of fruiting, so it became an attraction for the species of fruiteating avifauna. In this case, the importance of habitat for avifauna species can be seen, i.e. although it was located between disturbed habitats, but if it can still provide carrying capacity, the site will certainly continue to be utilized by Loriculus pusillus for its survival.

\section{Crater}

Specifically, in Darajat, there was a unique habitat which is a crater. This crater still had the availability of feed and nesting locations for certain types of avifauna. One species found was a small fruit-eating bird with fairly small distribution, Macropygia emiliana, which was found in natural stands directly adjacent to the crater area. Other species found around the crater were Lanius schach, Pnoepyga pusilla, Aethopyga eximia, Dicaeum sanguinolentum, Dicaeum trochileum, Halcyon cyanoventris, Todiramphus chloris, and Psaltria exilis.

Species that have low adaptability will be vulnerable to disturbance and can suffer population decline. Usually, species that have low adaptability tend to have specific types of feed and other habitat needs. Because of the importance of the birds role, bird species and the sensitivity of their presence to habitat needs to be monitored as an evaluation of habitat quality.

In the current era of development, an indicator is needed to assess the effect of development or to assess the quality of an area. This assessment is important because it can provide an overview of an event or change in the environment and how to reduce the negative impact of a change. According to Young et al. (2013), the development of biological criteria using bird communities has become an important approach now for monitoring and assessing environmental quality. 


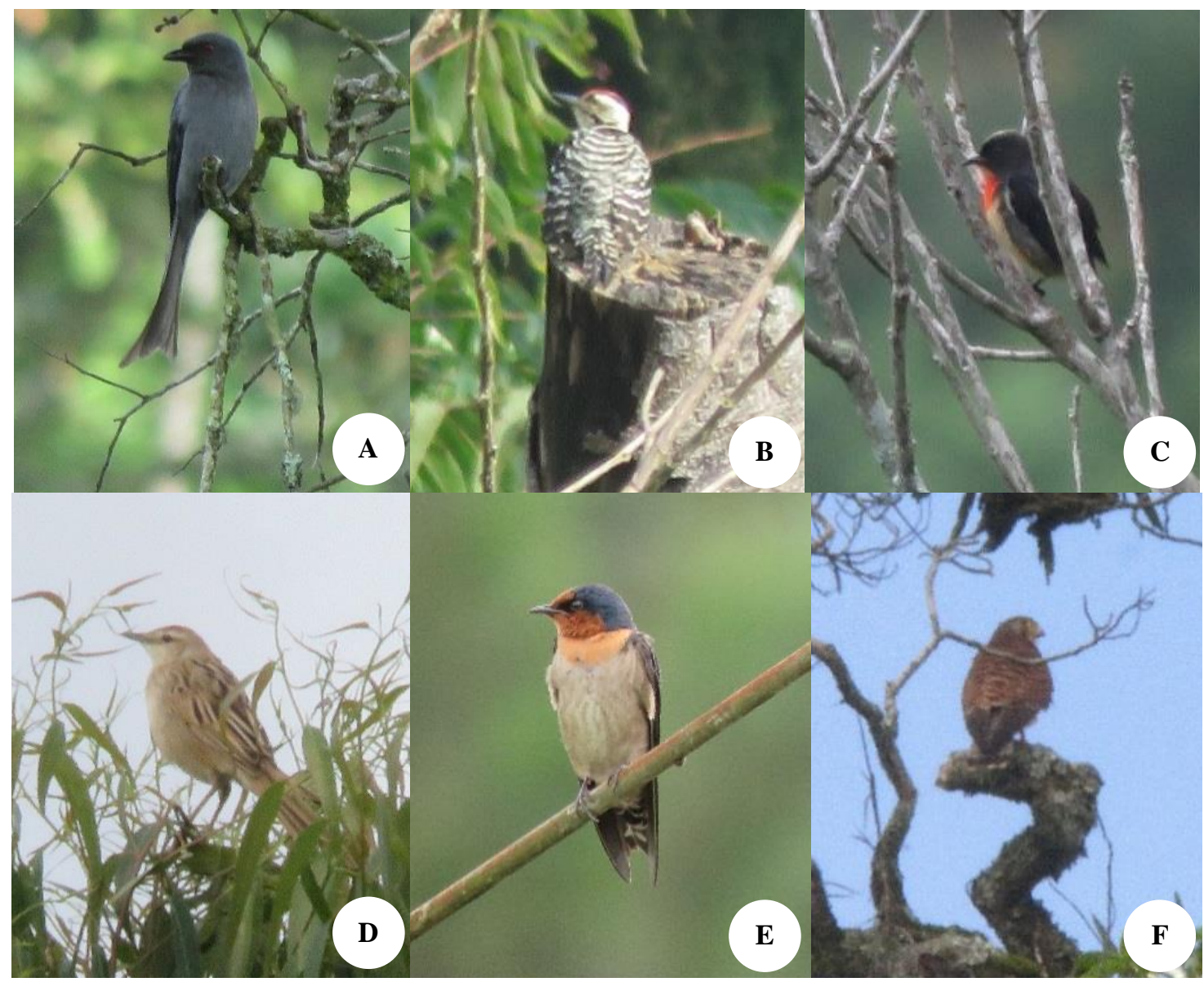

Figure 2. Some birds that found in geothermal power plant areas: A. Dicrurus leucophaeus, B. Dendrocopos moluccensis, C. Dicaeum sanguinolentum, D. Megalurus palustris, E. Hirundo tahitica, F. Falco moluccensis.Photographs by Syahrasf

According to Mariappan et al. (2013), the high diversity of birds is related to the more diverse plant species, which provide more choices for food preference, nesting and breeding places for birds. For example, tea agroforest arranged by shrubs, some clusters, and near the stream, is highly favorable by birds, but only if there are not many human activities. Wolf et al. (2012) said that vegetation structure of habitat is important and determines how many species could live in it. Vegetation cover is an important component of habitat and along with the surrounding landscape and management history might influence the composition of the breeding bird species.

In conclusion, based on the result of this study in three geothermal power plant areas in West Java, we conclude that the diversity of bird species is different from one location to others and it is affected by the type of vegetation. Gunung Salak had the highest diversity of bird species than any other locations with 105 species from 43 families. Species that were always found in all three locations were 49 species, including one species with Vulnerable status while the others had Least Concern status. There were two species with Endangered (EN) which only found in Gunung Salak. A total of 11 species were endemic species in which five were endemic in Java.
The presence of some birds was an indicator of ecosystem condition and functioning, including indicator of food chain, quality of water, quality of natural habitat, condition of riparian habitat, and the condition of open or disturbed habitat. Some birds were also associated with particular habitats, such as natural forests, riparians, a combination of natural forests and open habitats, tea plantations, and craters.

\section{ACKNOWLEDGEMENTS}

Acknowledgments were given to Centre Environment Sustainability Science (CESS), Padjadjaran University, Sumedang, Indonesia and the Management of Kamojang Indonesia Power-POMU, especially Budi Wibowo as GM. We also thank the surveyor team.

\section{REFERENCES}

Altaf M, Javid A, Khan AM, Khan MSH, Umair M, Ali Z. 2018. Anthropogenic impact on the distribution of the birds in the tropical thorn forest, Punjab, Pakistan. J Asia-Pac Biodiver 11 (1): 229-236. DOI 10.1016/j.japb.2018.03.001. 
Bibi F, Ali Z. 2013. Measurement of diversity indices of avian communities at Taunsa Barrage Wildlife Sanctuary, Pakistan. J Anim Plant Sci 23: 469-474.

Budiharta S, Erik Meijaard, DLA Gaveau, MJ Struebig, A Wilting, S Kramer-Schadt, J Niedballa, N Raes, M Maron, KA Wilson. 2018. Restoration to offset the impacts of developments at a landscape scale reveals opportunities, challenges and tough choices. Global Environ Ch 52: 152-161. DOI: 10.1016/j.gloenvcha.2018.07.008.

Budiharta S. 2010. Floristic composition at biodiversity protection area in Lubuk Kakap, District of Ketapang, West Kalimantan. Biodiversitas 11 (3): 151-156. DOI: 10.13057/biodiv/d110309.

Erniwati, Ervizal AMZ, Yanto S, Iswandi A. 2016. The value of secondary forest patches for bird conservation in palm oil landscapes of Riau, Sumatra. Biodiversitas. 17 (2): 791-798. DOI: 10.13057/biodiv/d170256.

Fiqa AP, F Fauziah, DA Lestari, S Budiharta. 2019. The importance of insitu conservation area in mining concession in preserving diversity, threatened and potential floras in East Kalimantan, Indonesia. Biodiversitas 20 (1): 198-210. DOI: 10.13057/biodiv/d200123.

Frederick P, Gawlik DE, Ogden JC, Cook MI, Lusk M. 2009. The White Ibis and Wood Stork as indicators for restoration of the everglades ecosystem. Ecol Indicat 9 (6): 83-95. DOI: 10.1016/j.ecolind.2008.10.012.

Khan MS, Aftab, Syed Z, Nawab A, Ilyas O, Khan A. 2013. Composition and conservation status of avian species at Hastinapur Wildlife Sanctuary, Uttar Pradesh, India. J Threaten Taxa 5 (12): 4714-4721.

Kosickia JZ, Chylarecki P. 2012. Effect of climate, topography, and habitat on species-richness of breeding birds in Poland. Basic Appl Ecol 13: 475-483. DOI 10.1016/j.baae.2012.07.007.

Krisanti AA, A Choirunnafi, NO Septiana, FW Pratama, F Amelia, A Manjaswari, PA Septiningtyas, AS Wati, JY Satria, LL Ani, T Wibowo, and Sugiyarto. 2017. The diversity of diurnal bird species on western slope of Mount Lawu, Java, Indonesia. Biodiversitas 18 (3): 1077-1083. DOI: 10.13057/biodiv/d180327.

Mariappan N, Ahamed Kalfan BK, Krishnakumar S. 2013. Assessment of bird population in different habitats of agricultural ecosystem. Intl $\mathbf{J}$ Sci Res Environ Sci 1 (11): 306-316.

Nursamsi I, Ruhyat P, Nurvita C, Hasna SM. 2018. Modeling the predicted suitable habitat distribution of Javan Hawk-eagle Nisaetus bartelsi in Java Island, Indonesia. Biodiversitas 19 (4): 1539-1551. DOI: $10.13057 /$ biodiv/d190447.

Partasasmita R, Atsuary ZIA, Husodo T. 2017. The use of forest canopy by various bird species in tropical forest montana zone, the Nature Reserve of Mount Tilu, West Java, Indonesia. Biodiversitas 18 (2):453-457.

Partasasmita R, Iskandar J, Malone N. 2016. Karangwangi peoples (South Cianjur, West Java, Indonesia) local knowledge of species, forest utilization and conservation. Biodiversitas 17 (1): 154-161. DOI: 10.13057/biodiv/d170123.

Philpott SM, Soong O, Lowenstein JH, Pulido AL, Lopez DT, Flynn DF, DeClerck F. 2009. Functional richness and ecosystem services: bird predation on anthropods in tropical agroecosystems. Ecol Appl 19 (7): 1858-1867.

Praptiwi RA, Ronna S, Tatang MS, Gusti W, Prawesti W, Jito S. 2019. Bird diversity in transition zone of Taka Bonerate, Kepulauan Selayar Biosphere Reserve, Indonesia. Biodiversitas 20 (3): 820-824.

Sekercioglu CH. 2012. Bird functional diversity and ecosystem services in tropical forests, agroforests and agricultural areas. J Ornithol 153 (1): 153-161.

Sudrajat S, MD Putro. 2019. The contribution of forest remnants within industrial area to threatened mammal conservation: A case study in liquefied natural gas industry in Bontang, Indonesia. Biodiversitas 20 (8): 2257-2265. DOI: 10.13057/biodiv/d200821.

Suryawan A, Isdomo Y, Anita M, Margaretta C. 2017. Identification of aves, herpetofauna, and arthropods in Miangas Island (Indonesia), the borderline of Wallacea ecoregion. Biodiversitas 18 (4): 1528-1537. DOI: $10.13057 /$ biodiv/d180331.

Teixeira PHR, Thel TN, Ferreira JMR, et al. 2014. Local knowledge and exploitation of the avian fauna by a rural community in the semi-arid zone of Northeastern Brazil. J Ethnobiol Ethnomed 10: 81. DOI: 10.1186/1746-4269-10-81

Wolf J, Baker R, Reed E. 2012. An assessment of vegetation cover for grassland bird breeding habitat in Southeastern Wisconsin. Bird Popul 11: $22-29$

Young JS, Ammon EM, Weisberg PJ, Dilts TE, Newton WE, Wong-Kone DC, Heki LG. 2013. Comparison of bird community indices for riparian restoration planning and monitoring. Ecol Indicat 34: 159164. 